American Journal of Infectious Diseases 7 (1): 20-23, 2011

ISSN 1553-6203

(C) 2011 Science Publications

\title{
Immunity to Diphtheria in Haemodialysis Patients
}

\author{
${ }^{1}$ Abdolreza Sotoodeh Jahromi, ${ }^{2}$ Mortaza Pourahmd, ${ }^{3}$ Sara Azhdari, \\ ${ }^{4}$ Gita Manshoori, ${ }^{5}$ Abdolhossain Madani and ${ }^{6}$ Seyed Hamid Moosavy \\ ${ }^{1}$ Department of Immunology, \\ ${ }^{2}$ Department of Infectious Diseases, \\ Research Center for Zoonosis Diseases, Jahrom University of Medical Sciences, Jahrom, Iran \\ ${ }^{3}$ Student Research Committee, Jahrom University of Medical Science, Jahrom, Iran \\ ${ }^{4}$ Department of Infectious diseases, Tehran University of Medical Science, Tehran, Iran \\ ${ }^{5}$ Department of Epidemiology, Center for determinants in health promotion, \\ Hormozgan University of Medical Science, Bandarabbas, Iran \\ ${ }^{6}$ Department of Internal Medicine, Hormozgan University of Medical Science, \\ Bandar Abbas, Iran
}

\begin{abstract}
Problem statement: The incidence of infectious diseases is increased in patients with chronic renal failure. Chronic renal failure severely influences the immune functions of the host. Diphtheria is of great epidemiological concern. Although mainly observed during childhood, unvaccinated adults and relatively immunocompromised patients are at increased risk for acquiring diphtheria. Approach: To evaluate the anti-Diphtheria immunity level in southern Iranian patients with end stage renal disease undergoing hemodialysis and to find its association with sex, age, blood hemoglobin, serum albumin and duration of dialysis. This cross sectional study was carried out on a total of 52 patients, who were on hemodialysis and 52 age and sex matched healthy individuals with without any underlying renal disease as a control group. Subjects in the both groups receiving anti-diphtheria toxoid vaccine or immunoglubins a year prior to the study were excluded. The serum anti-diphtheria IgG antibody levels were measured by an ELISA method. Results: Diphtheria protected individuals in the patients and the control groups were 34.6 and $63.30 \%$ respectively. Of the evaluating factors just hemodialysis duration found to affect on diphtheria immunity. Conclusion: Diphtheria protected individuals in the patients group were significantly less than diphtheria protected individuals in the control group $(\mathrm{p}=0.011)$. Hemodialysis duration has significant effect on anti-diphtheria immunity level.
\end{abstract}

Key words: Hemodialysis patients, chronic renal failure, control group, End-Stage Renal Disease (ESRD), Chronic Kidney Disease (CKD), toxoid vaccine, diphtheria toxoid

\section{INTRODUCTION}

Infectious diseases are the leading cause of death in End-Stage Renal Disease (ESRD) patients, second only to cardiovascular disease. They also contribute to a significant morbidity in patients with earlier stages of Chronic Kidney Disease (CKD) (Kausz and Gilbertson, 2006).

The incidence of infectious diseases is increased in patients with chronic renal failure (Laube et al., 2002). This is thought to be due to an immunosupressed status in this population (Litjens et al., 2008; Vacher-Coponat et al., 2008; Kaliuzhina et al., 2006). This is thought to be related to an impaired $\mathrm{T}$ cell activation by antigen presenting cells (Girndt et al., 1993; 1995), impaired immune Responses and Antigen-Specific Memory CD4+ T Cells (Litjens et al., 2008), defects in NK cell function (Vacher-Coponat et al., 2008), immunodeficiency status manifested decrease in the number of $\mathrm{CD} 3+, \mathrm{CD} 4+$ and $\mathrm{CD} 72+$ cells and phagocytosis intensification (Kaliuzhina et al., 2006), T and B-lymphocyte abnormalities and impaired responses to $\mathrm{T}$ cell dependent pathogens such as hepatitis B virus (Argani and Akhtarishojaie, 2006) and endothelial dysfunction (Hussein, 2010). Opportunistic infectious disease such as toxoplasmosis has more prevalence than healthy subjects (solhjoo et al., 2010).

Diphtheria is of great epidemiological concern. Although mainly observed during childhood, unvaccinated adults and relatively

Corresponding Author: Seyed-Hamid Moosavy, Department of Internal Medicine, Hormozgan University of Medical Science, Bandar Abbas, Iran 
immunocompromised patients are at increased risk for acquiring diphtheria (Karakus et al., 2007). Recent epidemiological studies indicate a low immunity to diphtheria in adults in industrialized countries and indicate that the number of insufficiently protected individuals has increased especially in the elderly (Kruger et al., 1999).

In 2004, WHO reported the incidence of diphtheria, tetanus and pertussis in Iran to be 6, 11 and 98 cases, respectively while in 2005 , the respective incidences were 15, 8 and 125 (WHO, 2006).

Vaccination is the most effective means of preventing infectious diseases. Immunization programs are of great importance in the prevention of infectious diseases in immunocompromised individuals. However, the immune response to various vaccinations is impaired in patients with chronic renal failure (Kruger et al., 1999), requiring multiple boosts to generate a robust protective response (e.g., diphtheria, pertussis and tetanus) (Storsaeter and Wolter, 2006).

The tetanus, diphtheria and pertussis vaccination programme in Iran has been running since 1950 using a local vaccine manufactured by Razi Institute (RaziDTwP), Tehran, Iran and the efficacy of the vaccine was confirmed by previous studies. These vaccinations have decreased the incidence and changed the epidemiology of these diseases (Zarei et al., 2007), but there is no routine vaccination for hemodialysis patients and So far only a few studies have focused on seroresponse to tetanus toxoid in these patients in Iran (Sagheb et al., 2009; Jahromi et al., 2009).

\section{MATERIALS AND METHODS}

Subjects: This cross sectional study was carried out on a total of 52 patients ( 36 men and 16 women), with a mean age of $57.75 \pm 14.26$ years, who were on hemodialysis therapy due to end-stage renal disease in the Hemodialysis Center of Jahrom University of Medical Sciences in 2010. Fifty two healthy individuals (35 men and 17 women) with normal serum levels for ceratinine and BUN and without any underlying renal disease with a mean age of $58.98 \pm 14.32$ years as a control group were enrolled in this research.

There were not significant differences between age $(p=0.912)$ and $\operatorname{sex}(p=0.112)$ in the cases and the controls.

As there were not data indicating past history of vaccination in both groups, the control group was randomly selected among the same community of patients group to increase the reliability of the results. Individuals in the both groups receiving anti-diphtheria toxoid vaccine or immunoglubins a year prior to the study were excluded.
Data collection: Data including sex, age, hemoglobin, serum albumin, duration of dialysis, Body Mass Index (BMI) were obtained from all of the hemodialysis patients and their medical records.

Serologic evaluations: Anti-diphtheria toxoid $\mathrm{IgG}$ level was determined on serum samples taken from patients before starting hemodialysis. Sera were separated and stored at $-70^{\circ} \mathrm{C}$ until analysis. Antibody levels were measured by commercial ELISA kits (IBLHamburg GmbH, Hamburg, Germany). Optical density was measured at $450 \mathrm{~nm}$ using ELISA reader (Awerness Instruments, USA). Based on the EPI Program of WHO, the assay cut-offs for protective level of tetanus antibody was set at 0.1 International Units (IU mL ${ }^{1}$ ) (Sagheb et al., 2009; (Olander et al., 2009). Concentrations above the assay cut-offs were considered to be seroprotective.

Ethics: The protocol of this study was approved by the ethics committee of Jahrom University of Medical Sciences.

Statistical analysis: Statistical analyses were performed using SPSS ver.11.5 software (SPSS Inc., Chicago, Illinois) Statistical differences of various clinical and laboratory parameters between groups were evaluated by Chi-Square or Mann-Whitney U tests. To compare the means of two groups, the two independent sample t-tests were used. p-values of less than 0.05 were considered as significant.

\section{RESULTS}

The mean serum anti-diphtheria IgG level of hemodialysis patients was $0.086 \pm 0.062$ and $0.613 \pm 0.725 \mathrm{IU} \mathrm{mL}^{-1}$ in the control group $(\mathrm{p}=0.001)$.

The patients and the control groups were divided into 3 groups by their anti-diphtheria IgG level.

Group 1: $\mathrm{IgG}<0.1 \mathrm{IU} \mathrm{mL}^{-1}$ which are not protective and need basic immunization through tetanus booster vaccine.

Group 2: $0.1<\operatorname{IgG}<1 \quad \mathrm{IU} \mathrm{mL}^{-1}$ who need to be controlled in 1-2 years and group 3: $1<\mathrm{IgG}<5 \mathrm{IU} \mathrm{mL}^{-1}$ who need to be controlled in 2-4 years. Therefore 34 $(65.40 \%)$ patients were not protected against diphtheria because their $\mathrm{IgG}$ less than $0.1 \mathrm{IU} \mathrm{mL}^{-1}$ (Cameron et al., 2009; Sagheb et al. 2009). Table 1 illustrates the characteristics of the patients and the control groups.

Among the contributing factors studied, only the patients with longer duration of hemodialysis had lower anti-diphtheria IgG level $(\mathrm{p}=0.021)$. 
Am. J. Infect. Dis., 7 (1): 20-23, 2011

Table 1: Comparison of the characteristics of patients and control groups

\begin{tabular}{llll}
\hline & Hemodialysis & & \\
Variables & patients & Control group & $\mathrm{p}$-value \\
\hline Age (years) & $57.75 \pm 14.26$ & $58.98 \pm 14.32$ & $\mathrm{p}=0.900$ \\
Gender & $36(69.23 \%)$ men & $35(67.30 \%)$ men & $\mathrm{p}=0.900$ \\
Anti-diph. IgG (IU mL1) & $16(30.77 \%)$ women & $17(32.70 \%)$ women & $\mathrm{p}=0.001$ \\
Level of protection & $0.086 \pm 0.062$ & $0.613 \pm 0.725$ & $\mathrm{p}=0.012$ \\
\hline
\end{tabular}

Table 2: Comparison of the characteristics of protected patients (group 2-3)

\begin{tabular}{llll}
\hline & Factors affecting & & \\
Anti-diphtheria & Level 2 (7 patients) & Level 3 (4 patients) & p-value \\
\hline Age (years) & $48.3 \pm 12.6$ & $51.4 \pm 10.8$ & 0.82 \\
BMI & $24.8 \pm 4.4$ & $25.2 \pm 4.7$ & 0.72 \\
Albumin $\left(\mathrm{g} \mathrm{dL}^{1}\right)$ & $4.3 \pm 0.68$ & $4.7 \pm 0.83$ & 0.43 \\
$\mathrm{Hb}\left(\mathrm{g} \mathrm{dL}^{1}\right)$ & $8.9 \pm 1.6$ & $8.5 \pm 1.3$ & 0.72 \\
Duration of & $28.75 \pm 22.35$ & $13.76 \pm 24.45$ & 0.03 \\
hemodialysis (weeks) & & & \\
\hline
\end{tabular}

It is also noteworthy that patients with the highest anti-diphtheria $\operatorname{IgG}$ and immunization against diphtheria (level 3) have the shortest duration of dialysis $(\mathrm{p}=0.03)($ Table 2$)$.

\section{DISCUSSION}

The immunodeficiency in patients with chronic renal failure makes them prone to more fatal outcomes of infectious diseases. A few studies were done on immunization against anti-diphtheria in patients with chronic renal failure (Kruger et al., 1999; Kreft et al., 2000; Sagheb et al., 2009).

Diphtheria protected individuals in the patients group were significantly less than tetanus protected individuals in the control group (21.15\% Vs $2.70 \%)$ (p $=0.011$ ).

Kruger reported $16 \%$ of patients were unprotected against diphtheria compared with $19 \%$ of the control group, his results and our results approximately are the same (Kruger et al., 1999).

The mean serum anti-diphtheria IgG level of the hemodialysis patients was significantly lower than the mean serum anti-diphtheria IgG level in the control group $(p=0.001)$. We found a significant negative effect by the duration of hemodialysis on antidiphtheria IgG level in our patients group $(p=0.03)$ and we did not find any significant effect by the other contributing factors on anti-diphtheria IgG level in either chronic hemodialysis or in the all hemodialysis patients. Also there was not statistically meaningful difference between the protected against diphtheria patients $(n=11)$ and those not protected $(n=41)$. The same results were showed by (Sagheb et al. 2009).
According to previous studies rapid decline in the titer of anti-diphtheria IgG in vaccinated hemodialysis patients who had a protected status after 6 months, among the different factors considered, only age significantly impaired or reduced the diphtheria immunity level (Kreft et al., 2000).

\section{CONCLUSION}

To conclude, it seems that most of our hemodialysis patients need booster or re-vaccination of diphtheria vaccine to increase their anti-diphtheria immunity. We recommend frequent monitoring of antibody levels after re-immunization against diphtheria in hemodialysis patients.

\section{ACKNOWLEDGEMENT}

This study was completely financed by Student Research Committee (SRC) of Jahrom University of Medical Sciences. The authors are grateful to the patients and the control individuals who accepted to enter this study. This article has been extracted from Ms azhdari's thesis.

\section{REFERENCES}

Argani, H. and E. Akhtarishojaie, 2006. Levamizole enhances immune responsiveness to intra-dermal and intra-muscular hepatitis $\mathrm{B}$ vaccination in chronic hemodialysis patients. J. Immun. Based Ther. Vaccines, 4: 3-6. PMID: 16734912

Cameron, C., J. White, D. Power and N. Crowcroft, 2009. Diphtheria boosters for adults: Balancing risks. Travel Med Infect Dis., 5:35-9. PMID: 17161317 
Girndt, M., H. Kohler, E. Schiedhelm-Weick, K.H.M. Zum Buschenfelde and B.T. Fleischer, 1993. Cell activation defect in hemodialysis patients: Evidence for a role of the B7/CD28 pathway. Kidney Int., 44: 359-365. PMID: 7690861

Girndt, M., M. Pietsch and H. Kohler, 1995. Tetanus immunization and its association to hepatitis B vaccination in patients with chronic renal failure. Am. J. Kidney Dis., 26: 454-460. PMID: 7645553

Hussein, F.S., 2010. Endothelial dysfunction induced by type 2 diabetes mellitus and fibrinolytic activity. Am. J. Biochem. Biotechnol., 6: 103-110. DOI: 10.3844/ajbbsp.2010.103.110

Jahromi, A.S., R. Raoofi, M. Sarikhani and A. Madani 2009. Evaluation of anti-tetanus immunity in haemodialysis patients. Am. J. Immunol., 5: 108112. DOI: 10.3844/ajisp.2009.108.112

Kaliuzhina, E.V., O.A. Geinits, V.V. Kaliuzhin and I.D. Pak, 2006. The condition of immune homeostasis in patients with chronic renal failure. Klin. Med., 84: 60-63. PMID: 17243614

Karakus, R., A.L. Aral, D.O. Kanat, K. Hizel and K. Caglar et al., 2007. Determinants of protection against diphtheria in adult hemodialysis patients. Ren Fail., 29: 829-834. PMID: 17994451

Kausz, A.T. and D.T. Gilbertson, 2006. Overview of vaccination in chronic kidney disease. Adv. Chronic Kidney Dis., 13: 209-214. PMID: 16815227

Kreft, B., A. Fischer, S. Kruger, K. Sack and H. Kirchner et al., 2000. The impaired immune response to diphtheria vaccination in elderly chronic hemodialysis patients is related to zinc deficiency. Biogerontology, 1: 61-66. PMID: 11707922

Kruger, S., M. Seyfarth, K. Sack and B. Kreft, 1999. Defective immune response to tetanus toxoid in hemodialysis patients and its association with diphtheria vaccination. Vaccine, 17: 1145-1150. PMID: 10195626
Laube, G.F., C. Berger, P. Goetschel, E. Leumann and T.J. Neuhaus, 2002. Immunization in children with chronic renal failure. Pediatr. Nephrol., 17: 638642. PMID: 12185473

Litjens, N.H., M. Huisman, M.V.D. Dorpel and M.G. Betjes, 2008. Impaired immune responses and antigen-specific memory CD4+ $\mathrm{T}$ cells in hemodialysis patients. J. Am. Soc. Nephrol., 19: 1483-1490. PMID: 18480314

Olander, R.M., K. Auranen, T. Harkanen and T. Leino, 2009. High tetanus and diphtheria antitoxin concentrations in Finnish adults--time for new booster recommendations? Vaccine, 27: 52955298. PMID: 19596410

Sagheb, M.M., S. Sajjadi and G. Sajjady , 2009. A study on the protection of hemodialysis patients against diphtheria and tetanus. Ren Fail., 31: 904909. PMID: 20030525

Solhjoo, K., A.S. Jahromi and A. Parnian-Rad, 2010. Anti-toxoplasma gondii antibodies in haemodialysis patients. Am. J. Infect. Dis., 6: 1317. DOI: 10.3844 ajidsp.2010.13.17

Storsaeter, J. and J. Wolter, 2006. Is there a need for a new generation of vaccines against pertussis. Expert Opin. Emerg. Drugs, 11: 195-205. DOI: 10.1517/14728214.11.2.195

Vacher-Coponat, H., C. Brunet, L. Lyonnet, E. Bonnet and A. Loundou et al., 2008. Natural killer cell alterations correlate with loss of renal function and dialysis duration in uraemic patients. Nephrol. Dial. Transplant., 23: 1406-1414. PMID: 18029366

WHO, 2006. Relevé épidémiologique hebdomadaire. Weekly Epidemiolog. Rec., 81: 197-208. http://www.who.int/wer/2006/wer8120.pdf

Zarei, S., M. Jeddi-Tehrani, M.M. Akhondi, H. Zeraati, T. Kheirkhah and M. Ghazanfari, 2007. Immunogenicity of a triple diphtheria-tetanuswhole cell Pertussis vaccine in Iranian preschool children. Iran J. Immunol., 4: 101-109. PMID: 17652850 